\title{
THE OORT LIMIT
}

\author{
J.J. BINNEY \\ Theoretical Physics \\ Keble Road, Oxford OX1 3NP, U.K.
}

\section{Introduction}

During galaxy formation non-baryonic dark matter is not expected to pack down into a thin disk. Hence the mass-to-light ratio $\Upsilon_{d}$ of the Galactic disk is a key number as the mass-to-light ratio of a purely baryonic system. If $\Upsilon_{\mathrm{d}}$ proves to be large, it suggests that the large values of $\Upsilon$ that are detected elsewhere in the Universe are due to dark baryonic matter rather than some exotic: form of matter. Conversely, a small value $\Upsilon_{d}$ lends credence to the idea that most of the mass in the Universe is non-baryonic by suggesting that baryonic systems are well endowed with luminous stars.

\section{Basic formulae}

In cylindrical polar coordinates $(R, \phi, z)$ Poisson's equation may be written

$$
4 \pi G \rho=\frac{1}{R} \frac{\partial v_{c}^{2}}{\partial R}-\frac{\partial K_{z}}{\partial z},
$$

where $v_{c}$ is the circular speed and $K_{z}$ is the vertical component of the gravitational field. The term $\partial v_{\mathrm{c}} / \partial R$ can be evaluated in terms of the Oort constants and proves to be negligible. The Jeans equation governing the vertical distribution of a tracer population of number density $\nu$ can be written

$$
\nu K_{z}-\frac{\partial}{\partial z}\left(\nu\left\langle v_{z}^{2}\right\rangle\right)=\frac{\partial}{\partial R}\left(\nu\left\langle v_{R} v_{z}\right\rangle\right)+\frac{\nu}{R}\left\langle v_{R} v_{z}\right\rangle
$$

Near the plane we expect that $\left\langle v_{R} v_{z}\right\rangle \simeq \alpha\left(v_{R}^{2}-v_{z}^{2}\right\rangle z / R$ with $0<\alpha<1$ and $\nu\left\langle v_{i}^{2}\right\rangle \sim \mathrm{e}^{-2 R / R_{\mathrm{d}}}$, so the r.h.s. of $(2)$ evaluates to $-\left(2 \alpha / R_{d}-1 / R\right)\left\langle v_{R}^{2}-v_{z}^{2}\right\rangle \nu z / R$. For plausible values of $\alpha$ and $R_{\mathrm{d}}$ $\left(\alpha=0.5, R_{\mathrm{d}}=R_{0} / 3\right)$ the ratio of the r.h.s of (2) to a typical term on its l.h.s. is r.h.s./l.h.s. $\sim h z / R_{0}^{2}$, where $h \simeq 300 \mathrm{pc}$ is the scale height of the disk. This argument suggests that the r.h.s. of (2) can be neglected. Combining the resulting opproximate equation with (1) and integrating the result through the disk we obtain successively

$$
4 \pi G \rho=-\frac{\partial}{\partial z}\left(\frac{1}{\nu} \frac{\partial}{\partial z}\left(\nu\left\langle v_{z}^{2}\right\rangle\right)\right) \quad \text { and } \quad 2 \pi G \Sigma(z) \simeq-\frac{1}{\nu} \frac{\partial}{\partial z}\left(\nu\left\langle v_{z}^{2}\right\rangle\right)
$$

where $\Sigma(z)=\int_{-z}^{z} \mathrm{~d} z \rho$.

The bad news is that these equations require differentiation of the measured quantities $\nu$ and $\left\langle v_{z}^{2}\right\rangle$. In the approximation in which motions in and perpendicular to the plane are decoupled, the good news is that both $\nu(z)$ and $\left\langle v_{z}^{2}\right\rangle(z)$ are determined by a single unknown function, the distribution of tracers with respect to vertical energy $E=\frac{1}{2} v_{z}^{2}+\Phi$. Kuijken \& Gilmore (1989) and Créze et al. (1997) exploit this connection: they adopt a parametric form of $\Phi(z)$ and use it and the measured form of $\nu(z)$ to determine $f(E)$, from which they can predict the distribution of velocities at any $z$. The parameters in $\Phi(z)$ are chosen to optimize the fit between the predicted and measured velocity distributions.

Kuijken \& Gilmore applied this technique to a sample of $512 \mathrm{~K}$-dwarfs that were identified in a photometric survey of fields near the SGP. Since such tracer stars lie in a cone with the Sun at 
the apex, there are few stars near $z=0$, and the sample cannot constrain $\rho_{0}$. Kuijken \& Gilmore (1991) concluded that the sample most srongly contrains

$$
\Sigma(1.1 \mathrm{kpc})=71 \pm 6 \mathrm{M}_{\odot} \mathrm{pc}^{-2} .
$$

The samples of Créze et al. are formed by stars with $M_{V}<2.5$ in the Hipparcos Catalogue. Depending on the distance out to which they include stars, their samples contain $3000-2300$ stars. Since they require $v_{z}$ but do not have radial velocities for most stars, their velocity data are confined to the proper motions of the $17 \%$ of the samples that lie at $|b| \leq 10^{\circ}$. They conclude that $\rho_{0}=0.076 \pm 0.015 \mathrm{M}_{\odot} \mathrm{pc}^{-3}$.

Bahcall (1984) and Bahcall, Flynn \& Gould (1992) chose rather to parametrize $f(E)$. Specifically, they assumed that the tracer population was isothermal: $f \propto \mathrm{e}^{-E / \sigma^{2}}$. Indeed, they assumed that every mass-bearing population is isothermal with some measured velocity dispersion $\sigma_{i}$. Then $\rho_{i}(z)=\Upsilon_{i} \nu_{i}(z)=\rho_{i 0} \mathrm{e}^{-\Phi / \sigma_{i}^{2}}$ and $\Phi$ has to solve the non-linear o.d.e.

$$
\frac{\mathrm{d}^{2} \Phi}{\mathrm{d} z^{2}}=4 \pi G \sum_{i} \rho_{i}=4 \pi G \sum_{i} \rho_{i 0} \mathrm{e}^{-\Phi / \sigma_{i}^{2}} .
$$

This equation was repeatedly solved for assumed values of the parameters $\rho_{i 0}$ until the predicted density law of a given tracer, $\nu_{i}(z)=\left(\rho_{i 0} / \Upsilon_{i}\right) \mathrm{e}^{-\Phi / \sigma_{i}^{2}}$, agreed with the measured density profile.

This technique was applied to old samples of $\mathrm{F}$ and $\mathrm{K}$ giants by Bahcall (1984), and to a new sample of $\mathrm{K}$ giants by Bahcall et al. (1992). In each study at least one population required an unexpectedly large value of $\Upsilon$. Different solutions $\Phi(z)$ were obtained depending on the population that was assigned the anomalous value of $\Upsilon$. Bahcall et al. (1992) concluded that

$$
\Sigma(1.1 \mathrm{kpc})=83 \mathrm{M}_{\odot} \mathrm{pc}^{-2} \text { and } \rho(0)=0.26 \mathrm{M}_{\odot} \mathrm{pc}^{-3} \text { with errors } \sim \pm 0.1 \mathrm{dex}
$$

This estimate of $\Sigma(1.1 \mathrm{kpc})$ agrees with that of Kuijken \& Gilmore within the errors, while the Bahcall et al. value of $\rho(0)$ is incompatible with that of Crezé et al. Since the Bahcall et al. samples, like those of Kuijken \& Gilmore, are confined to small patches of the sky, while those of Crezé et al. are all-sky, the newer value, $\rho(0) \simeq 0.076 \mathrm{M}_{\odot} \mathrm{pc}^{-3}$ is surely to be preferred.

\section{Estimating $\Upsilon_{\mathrm{d}}$}

Once $\Sigma(1.1 \mathrm{kpc})$ has been estimated, two things remain to be done before $\Upsilon_{d}$ can be evaluated: (i) estimate how much of $\Sigma(1.1 \mathrm{kpc})$ is attributable to the disk rather than any spheroidal halo; (ii) estimate the disk's surface luminosity density $L_{\mathrm{d}}$.

Kuijken \& Gilmore argued that, near $z=1.1 \mathrm{kpc}, \Phi$ could be approximated by the first two terms in its Maclaurin series: $\Phi(z)=K z+F z^{2}$. Physically, $K$ quantifies the mass within $|z|$ of the plane while $F$ quantifies the equatorial halo density. The Kuijken \& Gilmore sample imposes a single constraint on $K$ and $F: K_{z}=K+2 z_{0} F \simeq$ const. with $z_{0} \simeq 1.1 \mathrm{kpc}$. If the halo were spherical, the Galaxy's circular-speed curve would impose a second constraint: $0.094 K+F \times 1 \mathrm{kpc}=15 \pm$ $3 \mathrm{M}_{\odot} \mathrm{pc}^{-2}$. Combining these constraints Kuijken \& Gilmore concluded that $\Sigma_{\mathrm{d}}=48 \pm 9 \mathrm{M}_{\odot} \mathrm{pc}^{-2}$.

Dehnen \& Binney (1997) reached an essentially identical conclusion by a rather different route: by an analysis of global Galactic mass models similar to that of Caldwell \& Ostriker (1981), they concluded that (4) implies that $\Sigma_{\mathrm{d}}=50 \pm 2 \mathrm{M}_{\odot} \mathrm{pc}^{-2}$ unless either $R_{\mathrm{d}}$ is unexpectedly short $\left(\leq R_{0} / 4\right)$ or the halo is highly flattened $(b / a \leq 0.3)$.

The interstellar medium is thought to contribute $\Sigma_{\mathrm{ISM}} \simeq 13 \mathrm{M}_{\odot} \mathrm{pc}^{-2}$ to the local surface density (Bahcall et al. 1992), so dynamical estimates of the local disk mass imply that stars contribute $\Sigma_{*} \simeq 37 \mathrm{M}_{\odot} \mathrm{pc}^{-2}$ to the local surface density.

From K-band SPACELAB data Kent et al. (1991) infer that the Galaxy's surface luminosity density at $R_{0}$ is $I\left(R_{0}\right)=70 \mathrm{~L}_{\odot} \mathrm{pc}^{-2}$ for a solar $K$-band absolute magnitude $M_{K}(\odot)=3.41$. By comparison, from $C O B E / D I R B E$ data Malhotra et al. (1996) find that the Galaxy has $M_{K}=$ -24.06 , or $L_{K}=9.7 \times 10^{10} L_{\odot}, 44 \%$ larger than the value $L_{K}=6.7 \times 10^{10} L_{\odot}$ inferred by Kent et al. Thus it seems unlikely that $I\left(R_{0}\right)$ is significantly smaller than $70 \mathrm{~L}_{\odot} \mathrm{pc}^{-2}$. In view of the estimate given above of $\Sigma_{*}$ we conclude that the local $K$-band mass-to-light ratio is $\Upsilon_{K} \lesssim 0.52$.

Bruzual \& Charlot (1993) estimate that a system in which the star-formation rate declines exponentially with time constant $\tau=5 \mathrm{Gyr}$ and the IMF has Salpeter's form down to $0.1 \mathrm{M}_{\odot}$, has 


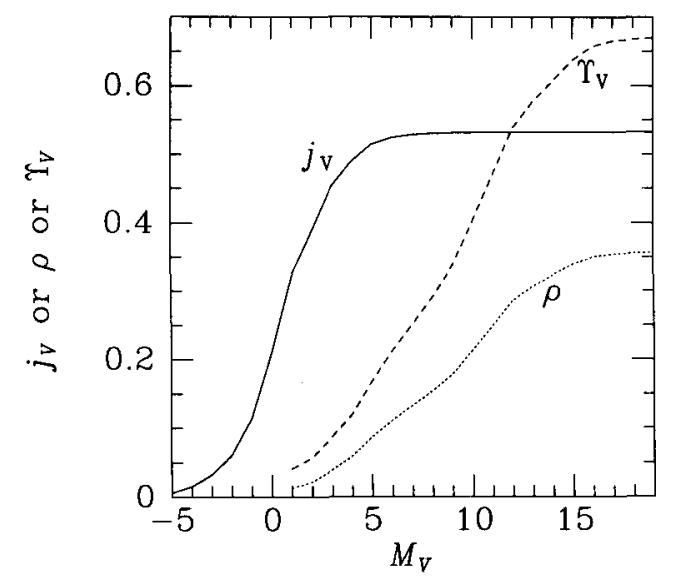

Figure 1. The full curve shows the $V$-band luminosity (in $10^{-1} L_{\odot} \mathrm{pc}^{-3}$ ) in stars brighter than $M_{V}$. The dotted curve shows the mass (in $10^{-1} \mathrm{M}_{\odot} \mathrm{pc}^{-3}$ ) in these stars. The dashed curve shows the corresponding values of $\Upsilon_{V}$.

$\Upsilon_{K} \simeq 0.74$ after $8 \mathrm{Gyr}$ and $\Upsilon_{K}=0.87$ after $12 \mathrm{Gyr}$. Thus the local mass-to-light ratio is lower than a Salpeter IMF implies, and the IMF should flatten significantly above $0.1 \mathrm{M}_{\odot}$.

Recently Gould et al. (1996) used HST to determine the luminosity function and the vertical density profile of low-mass stars. Their luminosity function agrees with that of Stobie et al. (1989) in turning down sharply faintward of $M_{V}=12$, with the result that they concluded that $M$ stars contribute only $\Sigma_{M} \simeq 13.5 \pm 3 \mathrm{M}_{\odot} \mathrm{pc}^{-2}$ to the local surface density. When this figure is added to the surface density in stars other than $\mathrm{M}$ stars, $\sim 15 \mathrm{M}_{\odot} \mathrm{pc}^{-2}$, we obtain $\Sigma_{*} \simeq 28.5 \mathrm{M}_{\odot} \mathrm{pc}^{-3}$, which is smaller than the corresponding dynamical estimate.

The luminosity functions of Stobie et al. and Gould et al. both rely on photometric distances. At $M_{V}>12$ luminosity functions that are based on trigonometric parallaxes of high-proper-motion stars lie consistently above those based on photometric parallaxes and therefore predict that there is more mass in M stars. For example, the luminosity function that Jahreiß and Wielen (1997) obtain from Hipparcos parallaxes of stars within $25 \mathrm{pc}$ of the Sun is flat from $M_{V} \simeq 12$ to $M_{V} \simeq 16.5$. When this luminosity function is combined with older data for luminous stars and the main-sequence mass-luminosity relation of Kroupa et al. (1993), one finds that stars brighter than $M_{V}=18$ have a cumulative mass-to-light ratio $\Upsilon_{V}=0.62$ - see Fig. 1. Observations of external galaxies that are similar to our own (de Jong 1996) suggest that the local disk has $V-K \simeq 2.8$. If the disk is this red, then $\Upsilon_{V}=0.62$ corresponds to $\Upsilon_{K}=0.17$, which is smaller even than the work of Malhotra et al. would suggest. Hence even with the larger number of low-mass stars that are found by parallax-based luminosity functions, the local mass-to-light ratio is surprisingly small.

In summary: estimates of the local luminosity surface density combined with dynamical estimates of the local mass surface-density yield a mas-to-light ratio $\Upsilon_{K}$ that is smaller than expected from a population of appropriate age with Salpeter IMF right down to $0.1 \mathrm{M}_{\odot}$. On the other hand, counts of faint stars yield an even smaller value of $\Upsilon_{K}$ than is observed. This suggests that there are more low-luminosity stars than even parallax-based surveys find, but fewer than a Salpeter IMF predicts.

\section{References}

Bahcall J.N., 1984, ApJ, 287, 926

Bahcall J.N., Flynn C., Gould A., 1992, ApJ, 389, 234

Caldwell J.A.R., Ostriker J.P., 1981, ApJ, 251, 61

Créze M., Chereul E., Bienaymé O., Pichon C., 1998, A\&A submitted

Dehnen W., Binney J.J., 1997, MNRAS in press

de Jong R.S., 1996, A\&A, 313, 377

Gould A., Bahcall J.N., Flynn C., 1996, ApJ, 465, 759

Jahreiß, H., Wielen, R., 1997, in "Hipparcos-Venice '97", ed. B. Battrick, ESA, Noordwijk, p. 675

Kent, S.M., Dame, T.M., Fazio, G., 1991, ApJ, 378, 131 
Kroupa, P., Tout C.A., Gilmore G., 1993 MNRAS, 262, 545

Kuijken K., Gilmore G., 1989, MNRAS, 239, 650

Kuijken K., Gilmore G., 1991, ApJL, L367, L9

Malhotra, S., Spergel D.N., Rhoads J.E., Li J., 1996, ApJ, 473, 687

Stobie R.S., Ishida K., Peacock J.A., 1989, MNRAS, 238709 